\title{
Building Blocks for Mobile Manipulation
}

\author{
Brian P. Gerkey \\ Willow Garage \\ 68 Willow Road, Menlo Park, California, USA \\ gerkey@willowgarage.com \\ http://www. willowgarage.com
}

\begin{abstract}
Following a long period of separate development, the twin fields of mobile robots and robotic manipulators have in recent years combined to form a new area of study, mobile manipulation. Research in mobile manipulation offers the promise of robots that can operate in workplaces and homes, with little or no modification, assisting people with everyday tasks. To realize that promise, there are significant scientific and engineering challenges to overcome.

In this talk, I will discuss how we, at Willow Garage, approach both the research and development of mobile manipulation. At the core of our approach are two engineering principles: (i) build reusable capabilities, and (ii) test what you build. While these are basic, even obvious, ideas in a development context, we apply them to great effect in our research projects. We make extensive use of simulation, building automated regression tests that run in simulation to verify the functionality of key capabilities (e.g., Fig. 1). We follow the maxim, "it's tested or it's broken."

I will also discuss the importance of openness in research, with a particular focus on sharing, validating, and reusing each others' work. By default, code that we write, notably the ROS infrastructure (http:// ros.org), is distributed under an open source license. By releasing the code that supports our published experimental results, and encouraging others to do the same, we aim to improve scientific practice in our field.
\end{abstract}
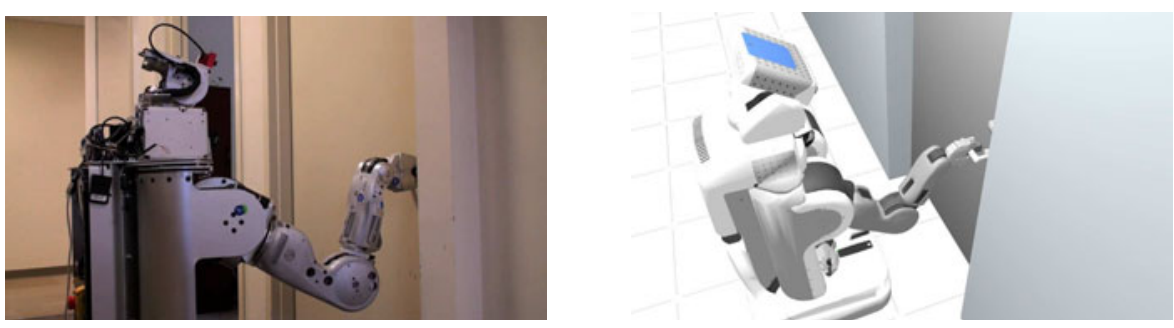

Fig. 1. Opening doors is a key capability. To ensure that the door-opening code will continue to work on the real robot (left), we test every change in simulation (right). 